\title{
Well leg compartment syndrome: an uncommon and devastating complication of advanced pelvic laparoscopic surgeries
}

Vimalakanthan Thanusan, Nishantha Liyanapthirana, Neville Dayawansa Perera

Department of Urology, National Hospital of Sri Lanka

Keywords: Laparoscopic pelvic surgeries; radical cystectomy; Lloyd Davis position; Trendelenburg position; fasciotomy

\section{Introduction}

Well, leg compartment syndrome[WLCS] is the development of acute compartment syndrome in the absence of trauma which may occur in a patient without any pre-existing vascular disease[1]. It is an uncommon complication, with dire consequences with loss of a limb in the failure of early diagnosis and prompt intervention. We present a 60 -year-old male who developed WLCS following a laparoscopic Radical cystectomy discussing the prevention, management and the outcome.

\section{Case presentation}

A 60-year-old male with non-muscle invasive high grade papillary transitional cell bladder carcinoma with synchronous right renal pelvic urothelial tumour [fig 1] underwent laparoscopic nephroureterectomy and radical cystectomy with ileal conduit urinary diversion. He was positioned in calf supported lithotomy position with elastic stockings and intermittent pneumatic compression. Operating time was $7 \frac{1}{2}$ hours with a head low tilt. Surgery was otherwise uneventful and managed at the ICU with epidural analgesia.

After ten hours he developed severe left calf pain followed by left leg swelling, restricted toe movements with decreased toe sensation, movements associated and severe pain on passive movements of the foot. Limb pulses were unaffected. A clinical diagnosis of compartment syndrome was made with rising creatinine kinase levels [26577 IU] after excluding the possibility of Deep Vein Thrombosis [DVT] with Doppler studies. Urgent four-compartment fasciotomy revealed that over $50 \%$ of peroneal and anterior compartment muscles were non-viable. which needed successive debridement procedures [Fig 2] and skin grafting for the wound closure. Residual defects included foot drop with a mild sensory

Correspondence: Vimalakanthan Thanusan

E-mail: thanusan18@gmail.com

(iDhttps://orcid.org/0000-0002-7645-0345

Received: 02-08-2020 Accepted: 18-12-202

DOI: http://doi.org/10.4038/sljs.v38i3.8669 deficit which was managed on standard orthopaedic guidelines and physiotherapy. Finally, he partially retained the ability to walk with residual neuralgic pain.

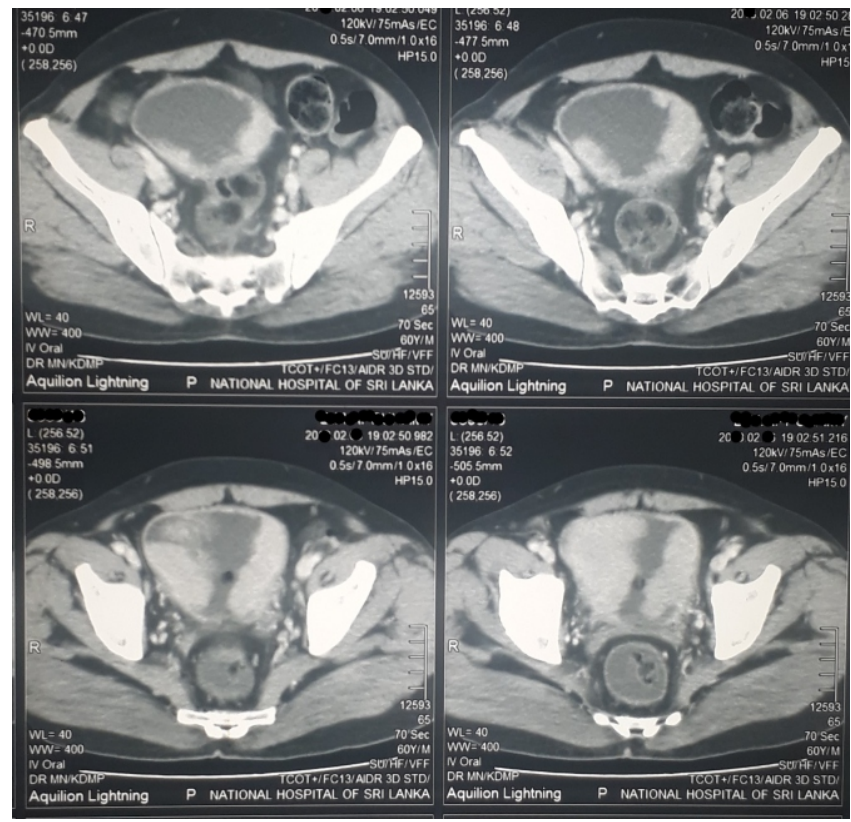

Figure 1. CT image of his bladder carcinoma

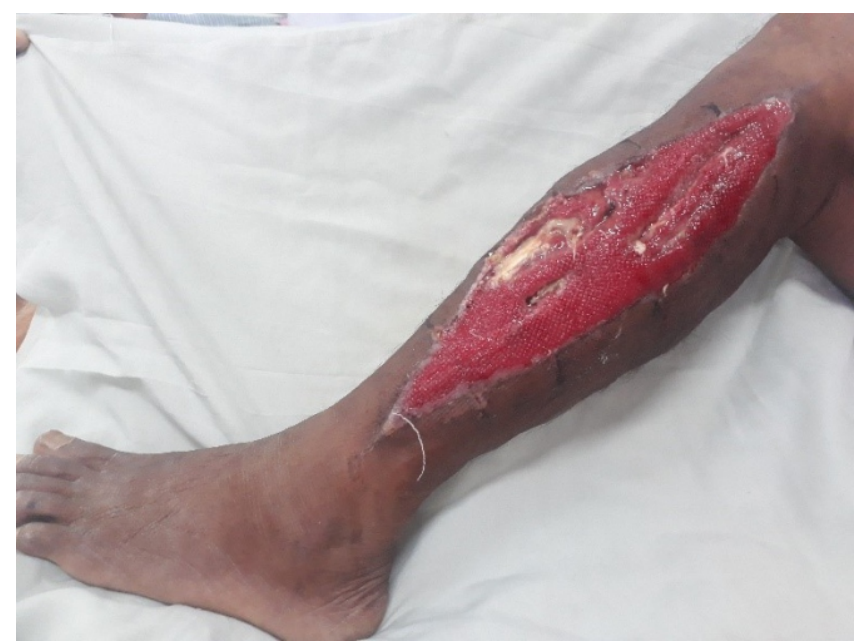

Figure 2. Fasciotomy wound upon recovery 


\section{Discussion}

WLCS was initially reported in the literature by Leff et al in 1979 after a prolonged urological surgery where the patient developed bilateral anterior compartment syndrome postoperatively[2]. Since then cases were reported in a wide range of surgical specialities such as orthopaedic, gynaecological, colorectal and $0.02 \%-0.03 \%$ of abdominopelvic surgeries using lithotomy, Lloyd Davis or Trendelenburg position. In cystectomies, the incidence rises from 1:3500 to 1:500 patients[1].

The underlying pathophysiology is mainly explained by the elevated compartmental pressure and hypoperfusion[1]. It is demonstrated that during prolonged periods of raised leg positions reduction any in systolic pressure below perfusion pressure causes ischaemia $[3,4]$. For each $2.5 \mathrm{~cm}$ elevation of the limb above the cardiac level leads to $2 \mathrm{mmHg}$ decrease in mean arterial pressure[3]. It is further enhanced by dorsiflexed ankles and head low position especially if it is beyond 15tilt. Increased compartmental pressure over $18 \mathrm{mmHg}$ has been reported during lithotomy position[1, 2, 4]. Subsequently, reperfusion injury-causing membrane injury, capillary leakage, oedema leads to a vicious cycle of ischaemia and increased compartment pressure characterised by pain disproportionate to the injury.

Risk factors can be patient-related or surgery-related factors. As mentioned earlier, patients with high BMI, young age and vascular diseases are more susceptible to this condition. Major intra-operative risk factors include the type of surgery and the specific position for a prolonged duration. Any abdominopelvic surgeries with leg elevation, knees and hips flexion are more susceptible.

Laparoscopic and robotic procedures are having a high likelihood of developing WLCS than open procedures due to the additional factor of reduced venous return associated with the pneumoperitoneum [1].

Risk has been shown to rise steeply for procedures extending over 4 hours and the median duration of surgery to develop this condition is reported as 7.5 as depicted in the present case[5].

In addition, focal compression with improperly placed stirrups and calf support also seems to influence the outcome. Meyer et al demonstrated that there is a favourable effect of $10 \mathrm{mmHg}$ decrease in compartmental pressure in heel supported position than calf support[4]. Intraoperative hypotension, circumferential wrappings and vasoactive drugs may also potentiate the occurrence of WLCS $[1,4]$.
As the complete recovery is unlikely in the majority of cases, proactive measures play a vital role in preventing this complication especially intraoperative concerns regarding the positioning. Periodical lowering of the limb from lithotomy position is recommended. Heel supported lithotomy position is more favourable than calf supported stirrups[4]. Whenever WLCS has anticipated it is advisable to use pharmacological anti-thromboembolic prophylaxis rather than rely on mechanical compression stockings.

As the diagnostic delay is an independent predictor of the severity of outcome, an early precise diagnosis is crucial to avoid reported complications which vary from minor sensorimotor deficit to loss of limb or life in a worst-case scenario [5].

A reliable clinical sign is calf pain especially upon passive stretching of the relevant compartment muscles. It is crucial to note that pain could be undermined by the postoperative epidural analgesia. Presence of paraesthesia or paresis is late features of neural ischaemia. It is important to note that limb pulses could be present up to the last phase which could give a false notion of security. Because of its relatively rare occurrence, often the possibility of WLCS is often clinically misdiagnosed as DVT. Epidural analgesia was also considered to be associated with delayed diagnosis due to its masking effect on pain, however, the evidence is still lacking. Elevation of serum creatinine kinase $[>2000 \mathrm{U} / 1]$ is supportive but should not delay the decisions taken on clinical grounds for decompression with four compartment fasciotomies to save the limb.

\section{Conclusion}

Awareness and early clinical detection are the keys to avoid this rare but detrimental vascular complication and the above case report highlights the importance of post-operative vigilant surveillance before the appearance of symptoms. As exemplified in the report, potential misdiagnosis as DVT and masking pain with epidural analgesia should be kept in mind which could cause delay the proper surgical treatment. Thus, it is important to highlight the need for monitoring against this in pelvic laparoscopic surgeries carrying a higher risk special perioperative measures should be carried out to prevent WLCS.

All authors disclose no conflict of interest. The study was conducted in accordance with the ethical standards of the relevant institutional or national ethics committee and the Helsinki Declaration of 1975, as revised in 2000 . 


\section{References}

1. Gill M, Fligelstone L, Keating J, Jayne DG, Renton S, Shearman $\mathrm{CP}$, et al. Avoiding, diagnosing and treating well leg compartment syndrome after pelvic surgery. The British journal of surgery. 2019 Aug;106[9]:1156-66. PubMed PMID: 31304580. Pubmed Central.https://doi.org/10.1002/bjs.11177

2. Leff RG, Shapiro SR. Lower extremity complications of the lithotomy position: prevention and management. The Journal of urology. 1979 Jul;122[1]:138-9.

https://doi.org/10.1016/S0022-5347[17]56290-4

3. Enderby GE. Postural ischaemia and blood-pressure. Lancet. 1954 Jan 23;266[6804]:185-7.

https://doi.org/10.1016/S0140-6736[54]91258-1
4. Meyer RS, White KK, Smith JM, Groppo ER, Mubarak SJ, Hargens AR. Intramuscular and blood pressures in legs positioned in the hemilithotomy position : clarification of risk factors for well-leg acute compartment syndrome. The Journal of bone and joint surgery American volume. 2002 Oct;84[10]:182935. https://doi.org/10.2106/00004623-200210000-00014

5. Christoffersen JK, Hove LD, Mikkelsen KL, Krogsgaard MR. Well Leg Compartment Syndrome After Abdominal Surgery. World journal of surgery. 2017 Feb;41[2]:433-8. https://doi.org/10.1007/s00268-016-3706-8

\section{Learning Points:}

- All the surgeons and anaesthetists involved in major laparoscopic pelvic surgeries, especially in Lloyd Davis and lithotomy positions should be more vigilant towards well leg compartment syndrome.

- Diagnosis can be made on clinical grounds and immediate decompression is mandatory in this limb/life-threatening emergency.

- Proactive intraoperative measures like preventing intraoperative hypotension and proper care in positioning are simple but efficient steps in avoiding this complication

- It should be kept in mind that the use of epidural analgesia and misdiagnosis of deep vein thrombosis could delay the diagnosis of well leg compartment syndrome. 